We plan to develop this service by training more volunteers and encouraging a 'buddy' system to review goals particularly around exercise/activity and weight loss.

\section{P-130 BEREAVED CAREGIVERS' EXPERIENCES OF END-OF-LIFE CARE FOR PEOPLE WITH ADVANCED HEART FAILURE}

Melanie Diggle, Sue Schutz, Dan Butcher. Oxford Brookes University, Oxford, UK

\subsection{6/spcare-2021-Hospice.147}

Background Heart failure is a life-limiting condition with a debilitating symptom burden (McMurray, Adamopoulos, Anker, et al., 2013). The prognosis for those living with heart failure is unpredictable and poor (Jaarsma, van der Wal, Hjelmfors, et al., 2018). Research has indicated that end-of-life care can improve symptom management and decrease caregiver burden (Buck, Harkness, Wion, et al., 2014; McIlfatrick, Doherty, Murphy, et al., 2018). However, despite this, referrals to palliative care services for people with heart failure remains low (Gadoud, Kane, Macleod, et al., 2014). Therefore, exploration of bereaved caregivers' experiences of providing end-of-life care to a person with heart failure is required.

Aim The aim of this review was to present and synthesise the current evidence around bereaved caregivers' experiences of providing end of life care for people with heart failure.

Methods A systematic review of the literature was undertaken using a search of four electronic databases (CINAHL, PubMed, BND, PsycINFO). Due to a scarcity of literature relating to heart failure, the search was expanded to include other long-term conditions. Research published between January 2000 - March 2021 with a qualitative methodology, focusing on bereaved caregivers' experiences of providing endof-life care for people with heart failure and other long-term conditions was included. Data was analysed and presented using a narrative synthesis approach.

Results Nineteen articles were critically appraised and included within this review. Four broad themes were identified: Limited and inadequate communication around the condition (including prognosis, preparations for death and the role of palliative care), the burden of caregiving, the positive experience of providing care at the end-of-life, limited provision of services and formal support.

Conclusion Bereaved caregivers experience significant challenges when caring for a loved one dying of heart failure or another long-term condition. This includes high levels of caregiver burden which is exacerbated by perceived inadequate communication, limited service provision and access to formal support. However, further research is required in this area to greater understand the specific end of life care experiences of bereaved caregivers of people with heart failure.

\section{P-131 HOW CONFIDENT DO PALLIATIVE AND NON-PALLIATIVE PATIENTS FEEL ABOUT MANAGING THEIR LYMPHOEDEMA?}

Olivia Core, Lucy Houghton, Annie Campbell. Dorothy House Hospice (Bath), Winsley, UK

\subsection{6/spcare-2021-Hospice. 148}

Background Lymphoedema is recognised as a chronic condition that requires lifelong management (National Lymphoedema Partnership, 2019). One of the NHS' core requirements for supporting individuals with a long term condition is promoting self-management, through professional led training, support and information sharing (NHS England, 2017). Clinical intervention is adapted to the patient needs, although often involves skin care, exercise, lifestyle and risk reducing advice, alongside the provision of a compression garment (British Lymphology Society, 2016).

Aim To explore palliative and non-palliative patient confidence with oedema self-management, via a clinical evaluation survey before and after a clinical intervention.

Methods New palliative and non-palliative patients attending the Dorothy House Hospice lymphoedema service between November 2020 and May 2021, received a questionnaire via post, or via their lymphoedema practitioner, for completion prior to initial assessment (Time 1), and at six weeks (palliative) or three months (non-palliative) follow-up (Time 2). Participants rated their confidence in self-managing their lymphoedema, and if swelling increased, on a five point Likert scale (Not at all confident-Very confident). Exclusion: re-referral of patient who had received previous lymphoedema input. Results 27 patients ( $n=15$ palliative) were invited to participate in the survey. Two patients declined participation. 25 patients took part (palliative $n=15$ \& non-palliative $n=10$ ). Complete data $\mathrm{n}=12$ participants. Incomplete data $\mathrm{n}=13$ $(\mathrm{n}=1$ declined participation during study, $\mathrm{n}=3$ misplaced forms, $\mathrm{n}=7$ died, $\mathrm{n}=2$ deteriorating health).

Self confidence in managing their lymphoedema

Non-palliative patients $[n=6]$ reported:

Not at all confident/Not confident - at $\mathrm{T} 1=58 \%$ \& at $\mathrm{T} 2=50 \%$,

Neutral [no change in confidence] at T1 \& T2=17\%,

Somewhat confident/Very confident at T1 $=25 \% \&$ at $\mathrm{T} 2=33 \%$. Palliative patients $[n=6]$ reported:

Not at all confident/Not confident - at T1 $=33 \% \&$ at $\mathrm{T} 2=8 \%$,

Neutral [no change in confidence] at T1 \& T2 $=25 \%$,

Somewhat confident/Very confident at $\mathrm{T} 1=42 \% \& \mathrm{~T} 2=67 \%$. Conclusion This small service evaluation suggests non-palliative patients are globally less confident about self-managing their lymphoedema than palliative patients pre- and post- our clinical intervention. Patients were generally more confident about their oedema management in stable and worsening conditions following clinical intervention. Further qualitative exploration with patients around their confidence in lymphoedema management, our clinical intervention and service delivery would be valuable.

\section{P-132 ADMINISTRATION OF SUBCUTANEOUS FLUIDS IN THE COMMUNITY SETTING; A QUALITY IMPROVEMENT PROJECT}

${ }^{1}$ Joy Ross, ${ }^{2}$ Stefan Rakowicz, ${ }^{1}$ Eva Trowers, ${ }^{2}$ Amrit Aujla, ${ }^{3}$ Amanda Rees, ${ }^{1}$ Emma Hall. ${ }^{1} S t$ Christopher's Hospice, London, UK; ${ }^{2}$ Bromleag Care Practice, Bromley, UK; ${ }^{3}$ Bromley Clinical Commissioning Group, Bromley, UK

10.1136/spcare-2021-Hospice. 149

Aim To identify patients suitable for subcutaneous fluid administration (SCF) in the home/care home setting and standardise clinical approach to assessment, decision making and evaluation of response.

Methods A standardised decision support tool was developed which supported bedside assessments. Patient/carer information leaflets were given and documentation of discussions/outcomes recorded in relevant clinical records (EMIS or SystmOne). We evaluated clinical response over three days: symptoms, side- 